\title{
Evaluation of Hospital-Based Acute Care Utilization by Uninsured Patients Enrolled in Free or Low-Cost Pharmacy Programs
}

\author{
Jessica Stickel, PharmD, (doctor of pharmacy candidate at the time of this work) ${ }^{1}$; Jennifer Kim, PharmD, BCPS, BCACP, BC-ADM ${ }^{2,3}$ \\ ${ }^{1}$ Novant Health New Hanover Regional Medical Center; ${ }^{2}$ University of North Carolina Eshelman School of Pharmacy, Chapel Hill; \\ ${ }^{3}$ Cone Health Internal Medicine Center, Area Health Education Center
}

\begin{abstract}
Background: Research is warranted to define the role of affordable pharmacy programs in optimizing healthcare utilization for uninsured patients. Methods: This was a pre-post study including uninsured patients from an internal medicine residency clinic who enrolled in free or low-cost pharmacy programs with clinical pharmacist support. Results: In the period following program enrollment $(N=116)$, there was a mean decrease of 0.23 acute care encounters (hospitalizations and emergency department [ED] visits) per patient ( $p=0.0210,95 \% \mathrm{Cl}$ 0.04-0.43). The mean decrease for hospitalizations was also statistically significant (0.17, $p=0.0052,95 \% \mathrm{Cl} 0.05-$ $0.28)$, but the mean decrease for ED visits was not $(0.06, p=0.3771,95 \% \mathrm{Cl}-0.08-0.21)$. Using the national average hospitalization cost of $\$ 10,700$, the decrease in hospitalizations represents an estimated savings of $\$ 246,100$. Conclusions: Enrollment in affordable pharmacy programs was found to be associated with decreased acute care encounters.
\end{abstract}

Keywords: uninsured, indigent care, hospital admissions, medication access, clinic-embedded pharmacist or primary care pharmacist

\section{Introduction}

In the United States, the Affordable Care Act helped to reduce the rate of uninsured individuals between 2013 and 2016, from $14.6 \%$ in 2013 to $8.6 \%$ in 2016 , but this population has since increased to $9.2 \%$ in $2019 .{ }^{1}$ The coronavirus pandemic is anticipated to lead to considerable income and insurance losses among those who were previously employed and/or insured, possibly exacerbating the trend seen from 2016-2019. ${ }^{2}$ Uninsured patients face significant barriers to medical care, including financial and nonfinancial obstacles. ${ }^{3}$ The majority of uninsured patients are low-income, and chronic disease management remains a primary concern for this population. ${ }^{3-5}$ As the consequences of poorly controlled chronic conditions include emergency department (ED) visits or hospital admission, the implications of managing chronic disease states extends beyond individual patient outcomes. ${ }^{6,7}$ The average hospitalization costs $\$ 10,700$, with the average ED visit costing $\$ 1,917 . .^{8,9}$ Acute care cost is generally left to the hospital system or larger community when patients are unable to pay, meaning both individual patients and society benefit from minimizing its use. .-11 $^{-11}$

\section{Corresponding author:}

Jennifer Kim, PharmD, BCPS, BCACP, BC-ADM

Cone Health Internal Medicine Center

Area Health Education Center

1200 North Elm Street Greensboro, North Carolina, 27401

Phone: 336-832-7885; Fax: 336-832-3925

Email: jen.kim@conehealth.com
Uninsured patients are disproportionately affected by lack of access to medications due to cost. ${ }^{12-15}$ With unreliable access to medications, adherence to treatment is compromised and overall disease state management is affected. ${ }^{16,17}$ Nonadherence to medications is often a precipitating factor before hospitalizations for chronic disease states. ${ }^{6,7,10,11,15}$ In a 2015 systematic review on medication adherence and health outcomes in chronic disease, reducing out-of-pocket costs for medications was shown to reduce blood pressure, cholesterol, incidence of myocardial infarction, and hospitalizations for lowincome patients. ${ }^{18}$ Another study found that reducing out-ofpocket expenses was as effective at improving adherence as more complex interventions including case management and patient education with behavioral support. ${ }^{19}$ Previous literature included a mixture of insured and under- or uninsured patients, but studies focusing on under- or uninsured patients can strengthen our understanding of how to support these vulnerable and growing populations.

Primary care clinics often lack the support necessary to reduce medication costs for patients. ${ }^{20}$ Pharmacists embedded in primary care clinics offer an opportunity to extend chronic disease management services and focus on interventions to improve medication affordability. ${ }^{21,22}$ Activities consist of clinical assessments and medication regimen optimization, including consideration for safety, efficacy, cost-effectiveness and adherence. Enrollment in prescription assistance programs reduces medication cost burden on patients, but the impact of these programs on acute care utilization is not well defined. ${ }^{23-}$ 25 This study aims to evaluate the association between enrollment in affordable pharmacy programs and hospitalbased acute care utilization (hospitalizations and ED visits) for uninsured patients. 


\section{Methods}

This retrospective pre-post study was reviewed and determined exempt by the Cone Health Institutional Review Board (study number 1459745-2). Participants in the study were patients from a suburban hospital-based internal medicine primary care clinic serving patients regardless of financial or insurance status. The clinic is a teaching site for resident physicians and interprofessional learners. Staff at the time comprised of a team including physicians, nurses, certified medical assistants, nurse technicians, phlebotomists, a dietician, a financial counselor, a social worker, and a clinical pharmacist. Of about 2,000 patients, coverage consisted of $45 \%$ Medicare, 17\% Medicaid, 19\% commercial, and 20\% were uninsured. More than half of the clinic population is estimated to have a household income at or below the Federal Poverty Level (FPL), approximately $70 \%$ of patients are black or African American, 25\% are white or Caucasian, 60\% are female, and $40 \%$ are male.

The pharmacist provided collaborative disease management for a variety of chronic conditions and screened referred patients for medication cost barriers to implement cost minimization interventions such as enrollment in affordable pharmacy programs. To qualify for these programs, the patient had to be uninsured with a household income at or below $200 \%$ of the FPL. One program was a state-wide free pharmacy (generic and brand medications available based on a formulary) for in-state patients that delivered medication to the patient's home. Another program was an on-site, clinic-associated pharmacy which provided reduced-cost medications (generic or brand depending on cost to the pharmacy based on 340B pricing) to patients who first met with the clinic financial counselor to confirm income and insurance eligibility. The prescriber confirmed this eligibility by contacting the financial counselor, then included a comment on each prescription, "internal medicine program" to notify the pharmacy of the patient's eligibility. The third program was an off-site pharmacy for patients within the county which provided reduced-cost generic medications and coordinated free access to brandname medications through manufacturer assistance programs.

A list of patients who visited the pharmacist during the study period of January 1, 2016 to June 30, 2019 was generated using the electronic health record (EHR). Patients were included in the study if they were 18 years or older, uninsured, and their pharmacy of record was one of the affordable pharmacy programs listed above. The EHR was reviewed to determine each patient's date of enrollment in one of the affordable pharmacy programs, marked as the index date. Multiple index dates could be recorded for the same patient if the date represented a unique access point into a pharmacy program, defined as enrollment into an alternate pharmacy program or re-enrollment after a period without access. An additional index date for the same patient was not recorded if the second index date was within 90 days of the first date. Charts were excluded if the pharmacy of record did not appear to be used (based on prescription fill history shown in the EHR, by contacting the pharmacy or by reviewing visit notes, if the index date was unclear, if the patient was enrolled in more than one of the three pharmacy programs at the same time, or if the index date was outside the defined study period.

After determining the index date for each enrollment, charts were reviewed to determine the number and type of acute care encounters (ED visits and hospitalizations) 90 days before and after each index date. Planned hospitalizations, defined as admissions for scheduled procedures, were excluded from review. All five hospitals within the health system were reviewed for acute care encounters.

The primary outcome was the mean number of acute care encounters (composite of hospitalizations and ED visits) per patient 90 days before and after enrollment into an affordable pharmacy program. Secondary outcomes included the components of the primary outcome and estimated cost savings associated with reduced acute care utilization.

The study was calculated to have $90 \%$ power with 100 index dates to detect a mean difference in the primary outcome of 0.2 encounters per patient with an effect size of 0.333 . Paired t-tests were used to analyze pre-post index date comparisons for normally distributed data, and descriptive statistics were used for all other data.

\section{Results}

Of 169 patients identified with 226 affordable pharmacy program enrollments, 116 patients with 139 index dates met study criteria for analysis. Twenty-seven patients were excluded for an index date outside of the study period; 26 patients were excluded due to not having a clear index date (i.e. lost to follow up and did not complete program enrollment process). Fifty-three percent $(n=74)$ of index dates indicated enrollment in the statewide free pharmacy, $24 \%(n=33)$ in the clinic-associated pharmacy, and 23\% $(n=32)$ in the off-site pharmacy (Table 1). Patients were 54\% ( $n=63)$ male, $62 \%$ $(n=72)$ African American, and $86 \%(n=100)$ spoke English as their primary language. All patients were diagnosed with at least one chronic condition, and the average age at index date was 51 years ( \pm 9.6 years).

A total of 94 acute care encounters (41 hospitalizations; 53 ED visits) were recorded before the index dates, with 62 acute care encounters (18 hospitalizations; 44 ED visits) after. There was a statistically significant decrease in the primary composite outcome, with a mean of $0.68( \pm 1.02)$ acute care encounters per patient before and $0.45( \pm 0.93)$ acute care encounters per patient after the index dates $(p=0.0210,95 \%$ confidence interval $[\mathrm{Cl}]$ 0.04-0.43). The mean difference for acute care encounters was $0.23( \pm 1.16)$ encounters per patient, leading to an effect size of 0.20 (Figure 1). 
While both hospitalizations and ED visits decreased following index dates, only hospitalizations had a statistically significant decrease. Before enrollment in the affordable pharmacy programs, there was a mean of $0.30( \pm 0.60)$ hospitalizations per patient; following the index dates, the mean number of hospitalizations per patient was $0.13( \pm 0.41)$, with a mean decrease of $0.17(p=0.0052,95 \% \mathrm{Cl} 0.05-0.28)$. For ED visits, there was a mean of $0.38( \pm 0.69)$ visits per patient before and $0.32( \pm 0.74)$ visits per patient after the index dates, leading to a non-significant mean decrease of $0.06(p=0.3771,95 \% \mathrm{Cl}$ 0.08-0.21). Before enrollment in the affordable pharmacy programs, the most frequent reasons for an ED visit and hospitalization were infection and cardiac complications, respectively. After enrollment, cardiac complications and gastrointestinal concerns tied as most common reasons for an ED visit, while a diabetic complication was the most common reason for hospitalization (Table $\mathbf{2}$ ).

The number of acute care encounters before and after the index dates varied based on the type of affordable pharmacy program (Figure 2). Acute care encounters for patients enrolled in the free pharmacy decreased by 46.3\%, from 54 (24 hospitalizations; 30 ED visits) to 29 (11 hospitalizations; 18 ED visits). Similarly, acute care encounters for patients enrolled in the clinic-associated pharmacy decreased by $47.8 \%$, from 23 (13 hospitalizations; 10 ED visits) to 12 ( 6 hospitalizations; 6 ED visits). Alternatively, the off-site pharmacy saw a $23.5 \%$ rise in acute care encounters following enrollment, with 17 encounters (4 hospitalizations; 13 ED visits) before and 21 encounters (1 hospitalization; 20 ED visits) after the index dates.

Overall, there were 23 fewer hospitalizations and 9 fewer ED visits in the 90 days following pharmacy enrollments compared with the 90 days before enrollment. With an average hospitalization in the United States estimated to cost $\$ 10,700,{ }^{8}$ twenty-three fewer hospitalizations equates to a savings of $\$ 246,100$. With an average ED visit costing $\$ 1,917,{ }^{9}$ nine fewer visits to the ED represents $\$ 17,300$ saved. In total, these decreases are associated with an estimated savings of $\$ 263,400$ in acute care spending 90 days post-enrollment (Figure 3).

\section{Discussion}

To our knowledge, this is the first study describing acute care utilization following patient enrollment in medication access programs by a primary care clinic-embedded pharmacist. Previous studies have demonstrated benefits of reducing medication costs and connecting uninsured patients with medication assistance programs, but many outpatient clinics do not routinely enroll patients in these programs. ${ }^{17,18,23-26}$ One review concluded that there is a lack of peer-reviewed literature assessing the impact of medication access on health outcomes. $^{27}$

After enrollment in free and low-cost pharmacy programs, hospitalizations decreased most for uninsured patients in this study (mean decrease of 0.17 hospitalizations per patient), with no statistically significant difference in ED visits (mean decrease of 0.06 visits per patient). Previous studies likewise demonstrated that increases in medication adherence can positively affect control of chronic conditions and decrease hospitalizations. ${ }^{11,18,26,28}$ The data concerning ED visits is less clear, however, with studies showing that medication nonadherence can lead to ED visits, but a lack of evidence demonstrating that decreasing medication costs is associated with decreased ED visits. ${ }^{25,26,29,30}$ One study examined rates of acute care utilization following facilitated patient enrollment in medication assistance programs facilitated by a social worker. ${ }^{26}$ There was a mean decrease of 0.096 acute care encounters per participant in the three months after enrollment, but the types of acute care encounters were not defined. More research is needed to define the relationship between decreased medication cost and ED visits.

Acute care encounters decreased by $46.3 \%$ after enrollment in the free pharmacy, but results varied among the two low-cost pharmacies. Providing medications free of charge has been advantageous in previous studies of patients lacking healthcare coverage, supporting the benefit found at the free pharmacy in our study. One study of indigent patients who were provided free medications for six months resulted in a $39.5 \%$ reduction in hospital admissions, similar to findings in our study. ${ }^{31}$ Another randomized trial found a decrease in blood pressure and increase in medication adherence following provision of free medications. ${ }^{32}$ Supplying free medications to patients without private insurance after myocardial infarction showed an increase in quality-adjusted survival and cost-effectiveness in a third study. ${ }^{33}$

While enrollment in the clinic-associated pharmacy led to similarly decreased acute care encounters after enrollment $(47.8 \%)$, enrollment in the off-site pharmacy was followed by a $23.5 \%$ increase in acute care encounters. Pharmacy location has been shown another factor of success in providing medications to uninsured or indigent patients, which may have played a role in these results. An article by Dent, et al. showed improved medication access for patients when a pharmacy was constructed at a community health center despite the sliding scale copays associated with prescriptions. ${ }^{34}$ In another study, on-site pharmacies positively affected patient retention and clinical outcomes when compared with off-site pharmacy use. ${ }^{35}$ This may have influenced the reduction in acute care encounters seen with the clinic-associated pharmacy in our study. Further studies would help to define optimal community pharmacy partnerships and how best to collaborate with offsite pharmacies.

The estimated decrease in healthcare costs associated with reduced acute care utilization in our study is consistent with previous studies examining cost benefits of interventions for uninsured or medically indigent patients. One prior study examined a case management intervention for uninsured 
patients and found a reduction in acute care utilization associated with a decrease of $\$ 6,667$ per patient in aggregate healthcare costs over the year. ${ }^{36}$ Another study reported an estimated $\$ 378,183$ savings to the hospital due to decreased inpatient admissions and outpatient visits after a six-month medication assistance program. ${ }^{31}$ The cost of drugs supplied was $\$ 27,588$, resulting in a net savings for the health system.

Positive outcomes of this study suggest an important role for clinic-embedded medication access services. Pharmacists, although underutilized in primary care practices, can help to improve multiple aspects of medication management. ${ }^{20,22,24}$ Studies have shown that pharmacists can enhance patient care quality, improve cost-effectiveness, reduce medication errors, and decrease provider burnout by lightening the burden of clinical and nonclinical duties. ${ }^{20,21,37}$ Shifting administrative tasks to pharmacy technicians can extend the pharmacist capacity and contribute to outcomes. ${ }^{38,39}$ The need to explore various strategies for medication access interventions and their impact on clinical outcomes has become increasingly critical due to the coronavirus-associated recession and growing uninsured population. ${ }^{2}$

Our study has several limitations. As data was collected by chart review, a direct assessment of medication access or adherence was not possible. Additionally, if patients sought care in a health system which does not interface with the study site EHR, their acute care encounters would not have been captured in this analysis. The extent of the association between enrollment in affordable pharmacies and a change in acute care use also cannot be determined, as we did not conduct a regression analysis. Confounding factors other than an improvement in medication access might have influenced our results, although there were no other initiatives implemented at the clinic during the study timeframe. At the time of this study, resources supporting robust research were lacking. In 2020, the health system data analytics enterprise was expanded to support higher quality studies. We hope our experience sparks more rigorous research to illuminate the relationship between medication access and acute care encounters.

The average acute care costs described for hospitalizations and ED visits are national estimates and may not necessarily reflect the patient- or hospital-specific costs in this study. Similarly, the drug costs associated with the prescriptions sent to the three pharmacy programs were unavailable; therefore, direct evaluation of overall healthcare expenditure saved through provision of free or reduced-cost medications was not performed. It is reasonable to suggest that costs associated with ED visits and hospital admissions offset those for the medications provided. Income information was not collected to due patients being referred to different programs for enrollment, but income level may influence outcomes.
In conclusion, there was an observed decrease in overall acute care encounters driven by hospitalizations after uninsured patients were enrolled in free and low-cost pharmacy programs. The results of this study suggest that increasing access to medications through affordable pharmacy options may help to reduce hospital-based acute care utilization, thereby reducing overall healthcare expenditure for uninsured patients. Future research is warranted to further define this relationship, the impact of affordable pharmacy programs, and the role of pharmacists in optimizing healthcare for uninsured patients.

Acknowledgements: Randy Absher, PharmD, BCPS

Funding/support: none

Conflicts of interest: none

Exemption granted by Institutional Review Board

\section{References}

1. Rosso RJ. U.S. health care coverage and spending. In Focus. https://fas.org/sgp/crs/misc/IF10830.pdf. Published January 2021. Accessed June 2021.

2. Agarwal SD, Sommers BD. Insurance coverage after job losses - the importance of the ACA during the Covid-associated recession. N Engl J Med. 2020;383:1603-1606.

3. Kamimura A, Panahi S, Ahmmad Z, et al. Transportation and other nonfinancial barriers among uninsured primary care patients. Health Serv Res Manag Epidemiol. 2018 Jan 5;5:2333392817749681.

4. Tolbert J, Orgera K, Singer N, et al. Key facts about the uninsured population. Kaiser Family Foundation. https://www.kff.org/uninsured/issue-brief/key-facts-aboutthe-uninsured-population/. Published December 2019. Accessed January 2020.

5. Wilper AP, Woolhandler S, Lasser KE, et al. A national study of chronic disease prevalence and access to care in uninsured U.S. adults. Ann Intern Med. 2008 Aug 5;149(3):170-6.

6. Kripalani S, Goggins K, Nwosu S, et al. Medication nonadherence before hospitalization for acute cardiac events. J Health Commun. 2015 Oct 9;20(0):34-42.

7. Ho PM, Rumsfeld JS, Masoudi FA, et al. Effect of medication nonadherence on hospitalization and mortality among patients with diabetes mellitus. Arch Intern Med. 2006 Sept 25;166(17):1836-41.

8. Frost A, Hargraves J, Rodriguez S. 2016 Health care cost and utilization report. Health Care Cost Institute, Washington, DC. https://healthcostinstitute.org/annual-reports/2016-healthcare-cost-and-utilization-report. Published January 2018. Accessed October 2019.

9. Torio CM, Moore BJ. Statistical Brief \#204. Healthcare cost and utilization project (HCUP). Agency for Healthcare Research and Quality, Rockville, MD. www.hcupus.ahrq.gov/reports/statbriefs/sb204-Most-ExpensiveHospital-Conditions.jsp. Published April 2016. Accessed October 2019.

10. Mongkhon P, Ashcroft DM, Scholfield CN, et al. Hospital admissions associated with medication non-adherence: a systematic review of prospective observational studies. BMJ Qual Saf. 2018 Nov;27(11):902-914. 
11. Sokol MC, Mcguigan KA, Verbrugge RR, et al. Impact of medication adherence on hospitalization risk and healthcare cost. Med Care. 2005 Jun;43(6):521-30.

12. Kennedy J, Morgan S. Cost-related prescription nonadherence in the United States and Canada: a system-level comparison using the 2007 International Health Policy Survey in Seven Countries. Clin Ther. 2009 Jan;31(1):213-9.

13. Gellad WF, Donohue JM, Zhao X, et al. The financial burden from prescription drugs has declined recently for the nonelderly, although it is still high for many. Health Aff (Millwood). 2012 Feb;31(2):408-16.

14. luga AO, Mcguire MJ. Adherence and health care costs. Risk Manag Healthc Policy. 2014 Feb 20;7:35-44.

15. Chima CC, Bruce MA, Pendergrass DB, et al. Strengthening the U.S. medication safety net by connecting abundance to need. Journal of Health Care for the Poor and Underserved. 2020 May;31(2):503-518.

16. Dustan HP, Caplan LR, Curry CL, et al. Report of the task force on the availability of cardiovascular drugs to the medically indigent. Circulation. 1992 Feb 1;85(2):849-60.

17. Patel MR, Piette JD, Resnicow K, et al. Social determinants of health, cost-related nonadherence, and cost-reducing behaviors among adults with diabetes: findings from the national health interview survey. Med Care. 2016 Aug;54(8):796-803.

18. Njie GJ, Finnie RK, Acharya SD, et al. Reducing medication costs to prevent cardiovascular disease: a community guide systematic review. Prev Chronic Dis. 2015 Nov 25;12:E208.

19. Viswanathan $M$, Golin $C E$, Jones CD, et al. Interventions to improve adherence to self-administered medications for chronic diseases in the United States: a systematic review. Ann Intern Med. 2012 Dec 4;157(11):785-95.

20. Hartkopf KJ, Heimerl KM, McGowan KM, et al. Expansion and evaluation of pharmacist services in primary care. Pharmacy. 2020 Jul 22;8(124):1-9.

21. Smith M, Bates DW, Bodenheimer T, et al. Why pharmacists belong in the medical home. Health Aff (Millwood). 2010 May;29(5):906-913.

22. Moreau $C$. The pharmacist as part of the primary care team during the COVID-19 pandemic. JABFM. 2021 February;34(S21-S25).

23. Zullig LL, Wolf $\mathrm{S}$, Vlastelica $\mathrm{L}$, et al. The role of patient financial assistance programs in reducing costs for cancer patients. J Manag Care Spec Pharm. 2017 Apr;23(4):407-411.

24. Johnson PE. Patient assistance programs and patient advocacy foundations: alternatives for obtaining prescription medications when insurance fails. Am J Health Syst Pharm. 2006 Nov 1;63(21 Suppl 7):S13-7.

25. Felder TM, Palmer NR, Lal LS, et al. What is the evidence for pharmaceutical patient assistance programs? A systematic review. J Health Care Poor Underserved. 2011 Feb;22(1):2449.

26. Burley MH, Daratha KB, Tuttle K, et al. Connecting patients to prescription assistance programs: effects on emergency department and hospital utilization. J Manag Care Spec Pharm. 2016 Apr;22(4):381-7.

27. Pharmacy Quality Alliance. Access to care: development of a medication access conceptual framework.

https://www.pqaalliance.org/assets/Research/PQA-Access-toCare-Report.pdf. Published March 2019. Accessed December $17,2020$.
28. Ruppar TM, Cooper PS, Mehr DR, et al. Medication adherence interventions improve heart failure mortality and readmission rates: systematic review and meta-analysis of controlled trials. J Am Heart Assoc. 2016 Jun 17;5(6):e002606.

29. Surbhi S, Graetz I, Wan JY, et al. Medication nonadherence, mental health, opioid use, and inpatient and emergency department use in super-utilizers. Am J Manag Care. 2020 Mar 1;26(3):e98-e103.

30. Blanchard J, Madden JM, Ross-Degnan D, et al. The relationship between emergency department use and costrelated medication nonadherence among Medicare beneficiaries. Ann Emerg Med. 2013 Nov;62(5):475-485.

31. Nykamp D, Ruggles D. Impact of an indigent care program on use of resources: experiences at one hospital. Pharmacotherapy. 2000 Feb;20(2):217-20.

32. Persaud N, Bedard M, Boozary AS, et al. Effect on treatment adherence of distributing essential medicines at no charge: the CLEAN meds randomized clinical trial. JAMA Intern Med. 2019 Oct 7;180(1):27-34.

33. Dhalla IA, Smith MA, Choudhry NK, et al. Costs and benefits of free medications after myocardial infarction. Healthc Policy. 2009 Nov;5(2):68-86.

34. Dent LA, Stratton TP, Cochran GA. Establishing an on-site pharmacy in a community health center to help indigent patients access medications and to improve care. J Am Pharm Assoc (Wash). 2002 May-Jun;42(3):497-507.

35. Lauer BR, Duggan JM, Eitniear L, et al. Use of an On-Site Outpatient Pharmacy for Acquisition of Antiretroviral Medications Compared to Off-Site Pharmacy Options: Impact on Retention in Care and Clinical Outcomes in People Living With HIV. J Pharm Pract. 2019 Aug 1;897190019866324.

36. Glendenning-Napoli A, Dowling B, Pulvino J, et al. Communitybased case management for uninsured patients with chronic diseases: effects on acute care utilization and costs. Prof Case Manag. 2012 Nov-Dec;17(6):267-75.

37. Awdishu L, Singh RF, Saunders I, et al. Advancing pharmacist collaborative care within academic health systems. Pharmacy (Basel). 2019 Oct 11;7(4):142.

38. Wheeler JS, Gray JA, Gentry CK, Farr GE. Advancing pharmacy technician training and practice models in the United States: Historical perspectives, workforce development needs, and future opportunities. Res Social Adm Pharm. 2020 Apr;16(4):587-590.

39. Ourth HL, Folstad J, Mambourg SE, et al. Evaluation of the potential impact of pharmacy technician performance of anticoagulation clinic tasks and operational efficiency. Am J Health Syst Pharm. 2019 Aug 15;76(16):1248-1253.

40. Schulman S, Kearon C. Definition of major bleeding in clinical investigations of antihemostatic products in non-surgical patients. J Thromb Haemost. 3(4):692-4. 
Table 1. Participant characteristics $(n=116)$

\begin{tabular}{|c|c|}
\hline Characteristic & No. (\%) \\
\hline Age, mean (SD) & 51.3 years $(9.6)$ \\
\hline \multicolumn{2}{|l|}{ Gender } \\
\hline Male & $63(54.3)$ \\
\hline Female & $53(45.7)$ \\
\hline \multicolumn{2}{|l|}{ Race/ethnicity } \\
\hline Black or African American & $72(62.1)$ \\
\hline White or Caucasian & $32(27.6)$ \\
\hline Other or two or more races & $11(9.5)$ \\
\hline Asian & $1(0.9)$ \\
\hline \multicolumn{2}{|l|}{ Primary language } \\
\hline English & $100(86.2)$ \\
\hline Spanish & $10(8.6)$ \\
\hline French & $2(1.7)$ \\
\hline Arabic & $2(1.7)$ \\
\hline Khmer & $2(1.7)$ \\
\hline \multicolumn{2}{|l|}{ Medical conditions ${ }^{a}$} \\
\hline Hypertension & $90(77.6)$ \\
\hline Hyperlipidemia & $63(54.3)$ \\
\hline Type II diabetes & $59(50.9)$ \\
\hline Depression & $36(31.0)$ \\
\hline Gastroesophageal reflux disease & $36(31.0)$ \\
\hline Asthma & $19(16.4)$ \\
\hline Chronic heart failure & $17(14.7)$ \\
\hline Obstructive sleep apnea & $14(12.1)$ \\
\hline Chronic obstructive pulmonary disease & $10(8.6)$ \\
\hline Atrial fibrillation & $9(7.8)$ \\
\hline \multicolumn{2}{|l|}{ Pharmacy enrollment $(n=139)$} \\
\hline Free pharmacy & $74(53.2)$ \\
\hline Clinic-associated pharmacy & $33(23.7)$ \\
\hline Off-site pharmacy & $32(23.1)$ \\
\hline
\end{tabular}

${ }^{a}$ Does not equal 100\%; multiple conditions possible for each patient 
Table 2. Primary diagnoses for acute care encounters

\begin{tabular}{|c|c|c|c|c|}
\hline \multirow{3}{*}{ Diagnosis } & \multicolumn{4}{|c|}{$\begin{array}{c}\text { Encounters } \\
\text { No. (\%) }\end{array}$} \\
\hline & \multicolumn{2}{|c|}{ ED visits } & \multicolumn{2}{|c|}{ Hospitalizations } \\
\hline & $\begin{array}{l}\text { Pre-enrollment } \\
(n=53)\end{array}$ & $\begin{array}{l}\text { Post- } \\
\text { enrollment } \\
(n=44)\end{array}$ & $\begin{array}{l}\text { Pre- } \\
\text { enrollment } \\
(n=41)\end{array}$ & $\begin{array}{l}\text { Post-enrollment } \\
(n=18)\end{array}$ \\
\hline Cardiac complication $^{\mathrm{a}}$ & $3(5.7)$ & $7(15.9)$ & $13(31.7)$ & $2(11.1)$ \\
\hline Infection $^{b}$ & $12(22.6)$ & $2(4.5)$ & $5(12.2)$ & $3(16.7)$ \\
\hline Respiratory complication ${ }^{c}$ & $4(7.5)$ & $2(4.5)$ & $8(19.5)$ & $2(11.1)$ \\
\hline Gastrointestinal concern $^{d}$ & $4(7.5)$ & $7(15.9)$ & $1(2.4)$ & $0(0)$ \\
\hline Musculoskeletal pain & $9(17.0)$ & $3(6.8)$ & $0(0)$ & $0(0)$ \\
\hline Diabetic complication $^{\mathrm{e}}$ & $1(1.9)$ & $3(6.8)$ & $4(9.8)$ & $4(22.2)$ \\
\hline Physical injury ${ }^{f}$ & $5(9.4)$ & $6(13.6)$ & $0(0)$ & $1(5.6)$ \\
\hline Migraine/headache & $1(1.9)$ & $2(4.5)$ & $2(4.9)$ & $1(5.6)$ \\
\hline DVT or PE & $1(1.9)$ & $1(2.3)$ & $3(7.3)$ & $0(0)$ \\
\hline Dental pain or infection & $2(3.8)$ & $3(6.8)$ & $0(0)$ & $0(0)$ \\
\hline Neurologic concern ${ }^{g}$ & $1(1.9)$ & $2(4.5)$ & $0(0)$ & $2(11.1)$ \\
\hline Alcohol use disorder & $4(7.5)$ & $1(2.3)$ & $0(0)$ & $0(0)$ \\
\hline Major bleeding ${ }^{\mathrm{h}}$ & $0(0)$ & $0(0)$ & $1(2.4)$ & $1(5.6)$ \\
\hline Clinically relevant non-major bleeding ${ }^{\mathrm{h}}$ & $1(1.9)$ & $3(6.8)$ & $1(2.4)$ & $0(0)$ \\
\hline Major depressive disorder & $1(1.9)$ & $0(0)$ & $0(0)$ & $1(5.6)$ \\
\hline Other, minor ${ }^{i}$ & $4(7.5)$ & $2(4.5)$ & $0(0)$ & $0(0)$ \\
\hline Other, major ${ }^{j}$ & $0(0)$ & $0(0)$ & $3(7.3)$ & $1(5.6)$ \\
\hline
\end{tabular}

Includes a chest pain/angina, chronic heart failure, coronary artery disease, mitral valve stenosis, hypertension, atrial fibrillation/flutter, ${ }^{b}$ sepsis, cellulitis, osteomyelitis, community-acquired pneumonia, urinary tract infection, bronchitis, influenza, viral syndrome, casthma, chronic obstructive pulmonary disease exacerbation, dyspnea, pulmonary edema, denteritis, nausea/vomiting/diarrhea, abdominal pain, ediabetic ketoacidosis, hyperglycemic hyperosmolar syndrome, hyperglycemia, hypoglycemia, diabetic foot wound, fracture/sprain, laceration, first degree burn, motor vehicle accident, etc, ssyncope, fall, seizure, abnormal gait, numbness, hInternational Society on Thrombosis and Haemostasis criteria ${ }^{40}$, idermatitis, dizziness, allergic reaction, tinnitus, acute urinary retention, jacute kidney injury, accidental phenytoin poisoning, symptomatic anemia, hypokalemia 
Figure 1. Average Number of Acute Care Encounters Per Patient

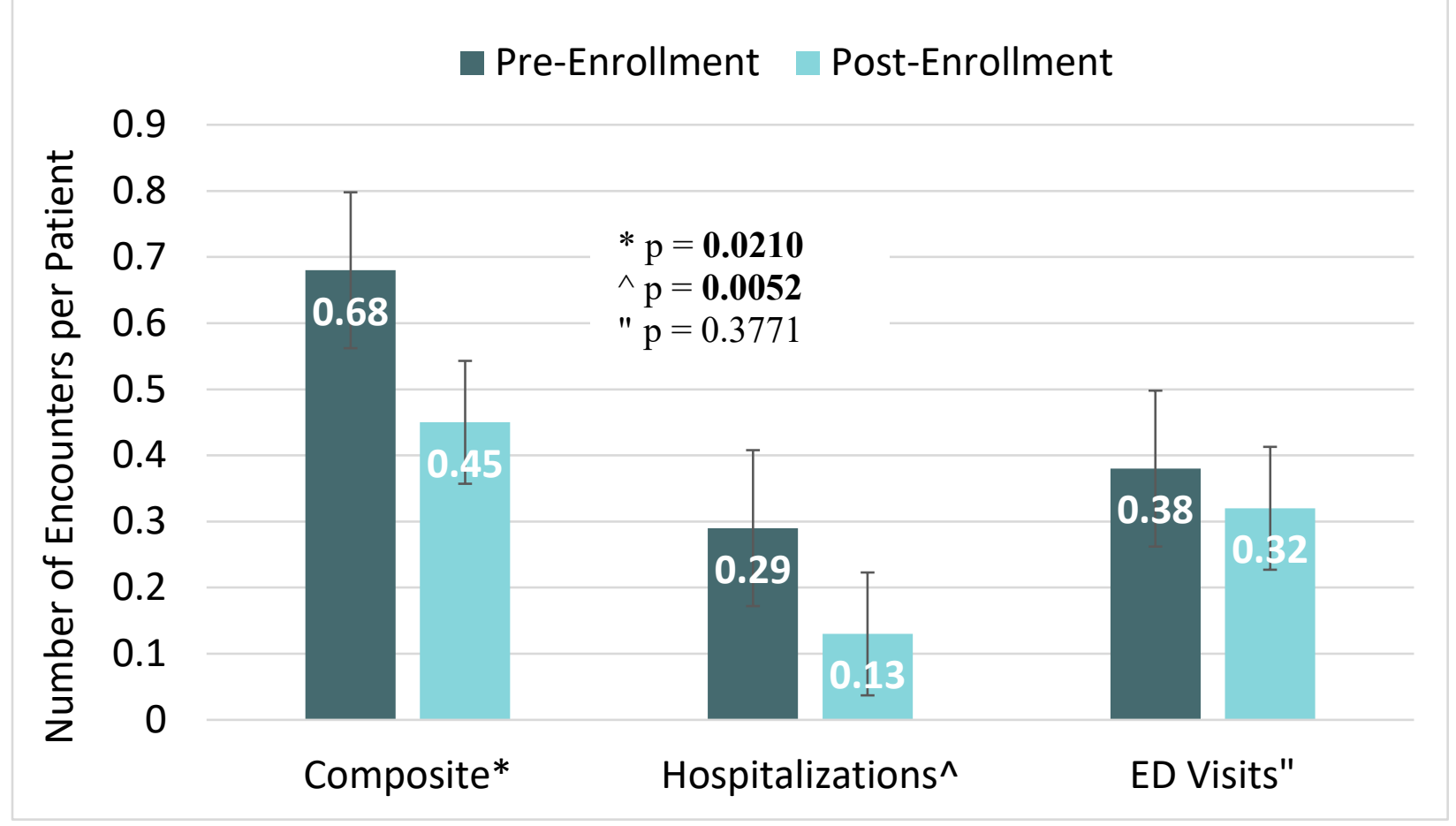

Figure 2. Number of Acute Care Encounters by Pharmacy Type

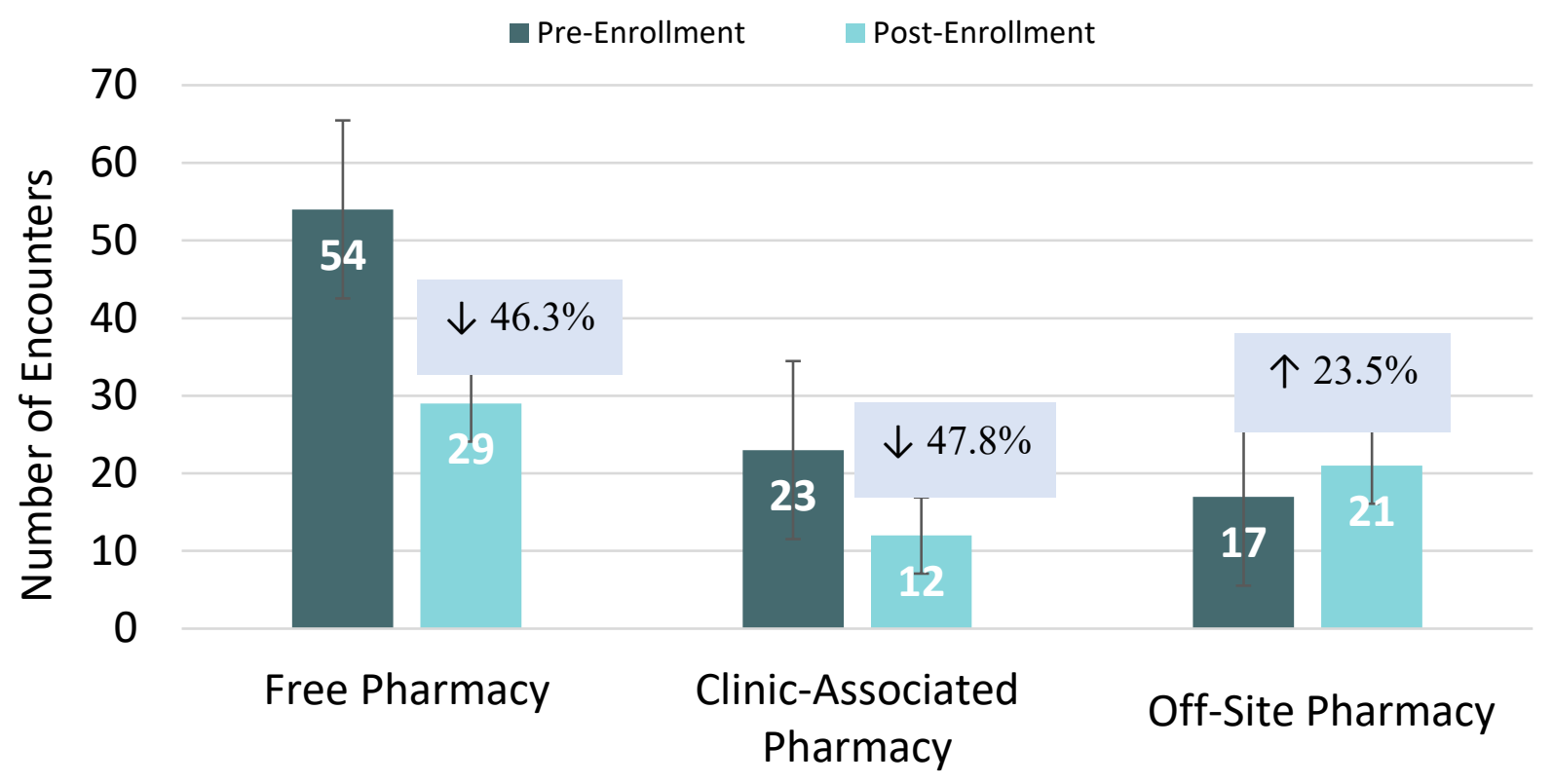


Figure 3. Estimated Cost of Hospital-Based Acute Care Encounters

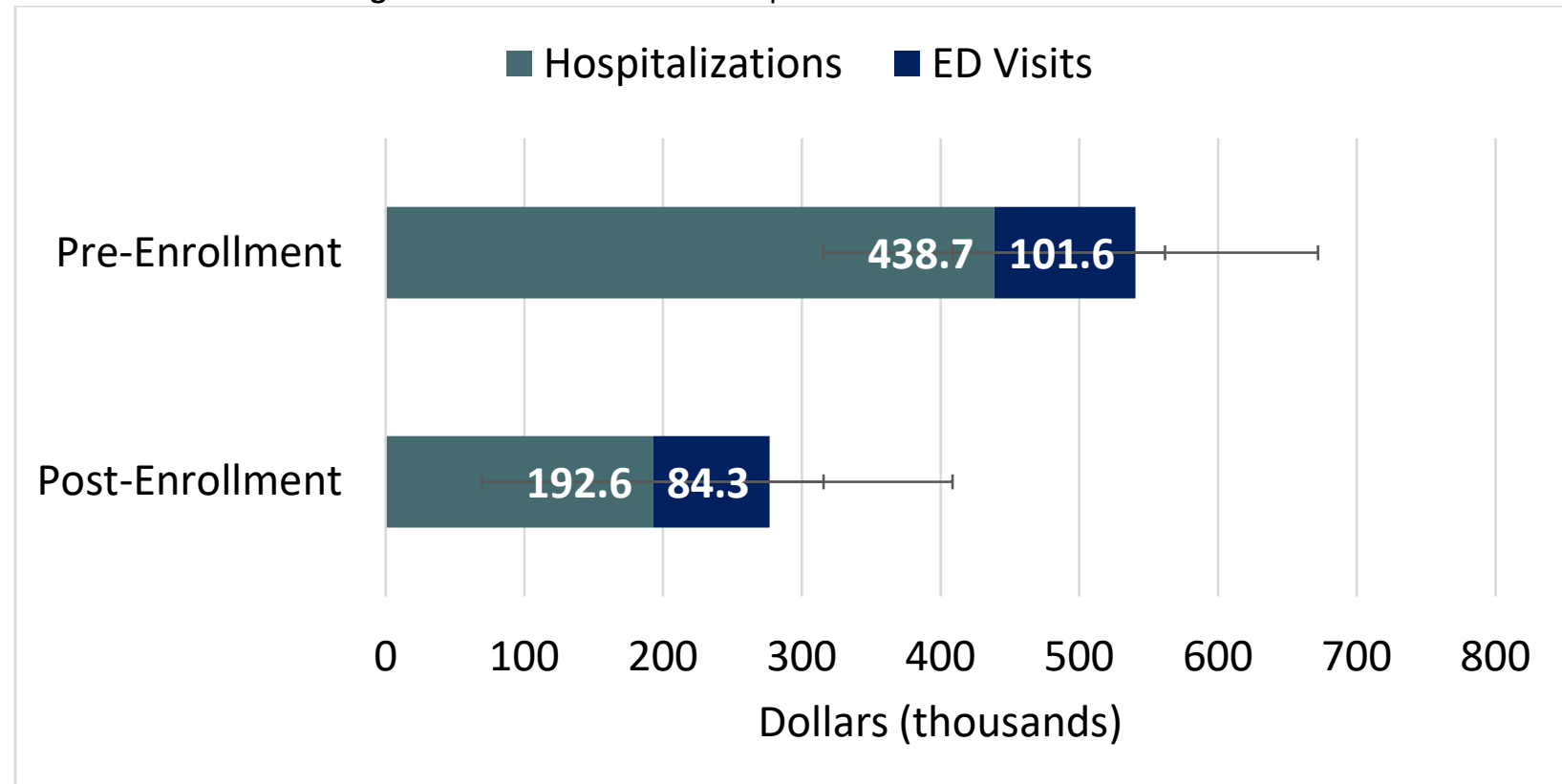

Source: Health Care Cost and Utilization Report ${ }^{8,9}$ 